\title{
FREQUENCY-WAVENUMBER DOMAIN FILTERING FOR IMPROVED DAMAGE VISUALIZATION
}

\author{
M. Ruzzene \\ School of Aerospace Engineering, Georgia Institute of Technology, Atlanta, GA 30332
}

\begin{abstract}
This paper presents a technique for the analysis of full wavefield data in the wavenumber/frequency domain as an effective tool for damage detection, visualization and characterization. Full wavefield data contain a wealth of information regarding the space and time variation of propagating waves in damaged structural components. Such information can be used to evaluate the response spectrum in the frequency/wavenumber domain, which effectively separates incident waves from reflections caused by discontinuities encountered along the wave paths. This allows removing the injected wave from the overall response through simple filtering strategies, thus highlighting the presence of reflections associated to damage. The concept is first illustrated on analytical and numerically simulated data, and then tested on experimental results. In the experiments, full wavefield measurements are conveniently obtained using a Scanning Laser Doppler Vibrometer, which allows the detection of displacements and/or velocities over a userdefined grid, and it is able to provide the required spatial and time information in a timely manner. Tests performed on a simple aluminum plate with artificially seeded slits simulating longitudinal cracks, and on a disbonded tongue and grove joint show the effectiveness of the technique and its potential for application to the inspection of a variety of structural components.
\end{abstract}

Keywords: Wavefield, Wavenumber/frequency domain, filtering.

PACS: 46.40.-f, 46.40.Cd, 46.80.+j, 07.10.-h.

\section{INTRODUCTION}

Among the various technologies explored for SHM, guided waves testing has shown great potentials [1,2]. The attractive features of guided waves include sensitivity to a variety of damage types, as well as the capability of traveling relatively long distances within the structure under investigation. Piezoelectric phased arrays have been proposed to focus guided waves and directly obtain information regarding damage direction [3] or to tune a specific wave mode in a multi-mode signal [4]. Phased arrays have been also proposed as directional filters to remove boundary reflections and directly identify the location of damage [5]. A similar objective is here pursued, without however the application of phased arrays. A Scanning Laser Doppler Vibrometer (SLDV) is used to measure the velocity of the inspected surface in points belonging to a predefined grid. Scanning the grid and post-processing the data allows the detection and the visualization of the full wavefield as it propagates in the structure. The resulting images describe the main features of the propagating wave and show its interactions with discontinuities that may be encountered along the wave path [6]. Damage can be thus immediately detected and localized through limited processing. Moreover, wavefield time-domain data can be fed to signal processing algorithms to obtain detailed images of the defect. For example, 
the application of multi-dimensional Fourier Transforms (FT)s in space and time provide the representation of the component response in the frequency/wavenumber domain. In this domain, all wave components propagating in directions opposite to the direction of propagation of the main injected pulse are clearly highlighted, so that the presence of reflections and mode conversions caused by the presence of damage are immediately noticeable [7]. The application of two-dimensional FT (2D FT) has previously been proposed in [8] for the analysis of multi-mode wave signals for the identification of dispersion relations and the characterization of various modes. In this work, the frequency/wavenumber representation is used to devise simple filtering strategies which are able to eliminate the applied excitation and corresponding propagating wave from the recorded response, while highlighting the presence of any reflections along the wave path.

\section{CONCEPT OF FREQUENCY/WAVENUMBER FILTERING}

\section{Application to a one dimensional propagating wave}

The basic concept behind the filtering technique under consideration is illustrated for a stress wave in a one dimensional (1D) waveguide. The considered configuration is depicted in Figure 1, and assumes the presence of a discontinuity in material and geometry at location $x=x_{0}$. The 1D stress in the region $x<x_{0}$ can be expressed as a sum of incident and reflected stress waves, according to the following expression:

$$
\sigma_{x}(x, t)=A_{0}\left[e^{i\left(\omega_{0} t-k_{0} x\right)}+\operatorname{Re}^{i\left(\omega_{0} t+k_{0} x-2 k_{0} x_{0}\right)}\right]
$$

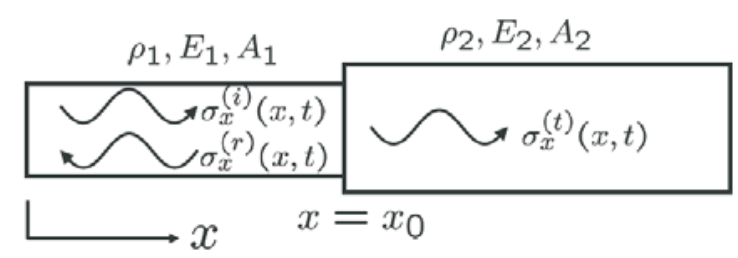

FIGURE 1: 1D waveguide.

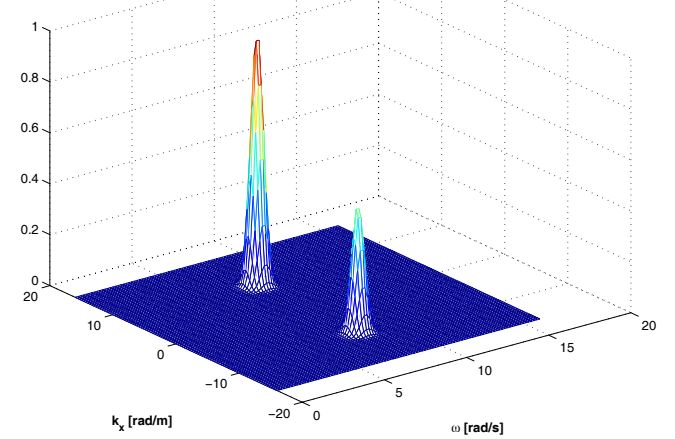

FIGURE 2: 2D FT of propagating stress wave.

where $A_{0}$ is the wave amplitude, while $\omega_{0}$ and $k_{0}$ respectively denote the wave frequency and wavenumber. It is assumed that both spatial and time information are available for the stress distribution in the domain of interest. Under this assumption, one can evaluate the two-dimensional Fourier Transform (2D FT) of $\sigma_{x}(x, t)$, whose magnitude features two peaks in the frequency/wavenumber domain at $\omega=\omega_{0}, k=k_{0}$, which correspond to incident and reflected waves (Figure 2). The 2D FT effectively separates the two wave components and allows the application of windowing functions to filter out one of the components. Upon filtering, the residual signal can be transformed back through an inverse FT to the space/time domain for visualization and further processing. This procedure is particularly useful for damage detection purposes, where reflected components carry information regarding the presence and the nature of damage. In many occasions, the reflections are 
small in amplitude and are often overshadowed by noise or by the incident wave. This makes the identification of damage, and its potential characterization difficult. From this perspective, 2D FT-based filtering represents an attractive approach to separate and highlight the presence of reflections in a given stress wave. The process is depicted graphically in Figures 3, 4, which respectively show a Hanning window overlapped to the signal's 2D spectrum and the residual signal upon filtering.

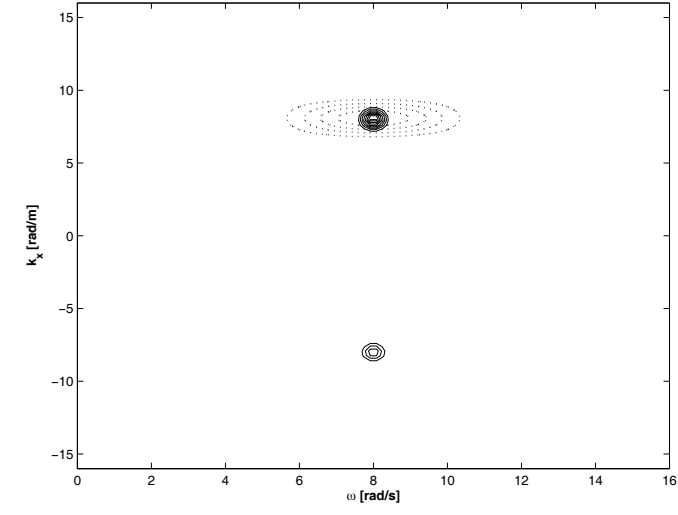

FIGURE 3: Windowed 2D FT.

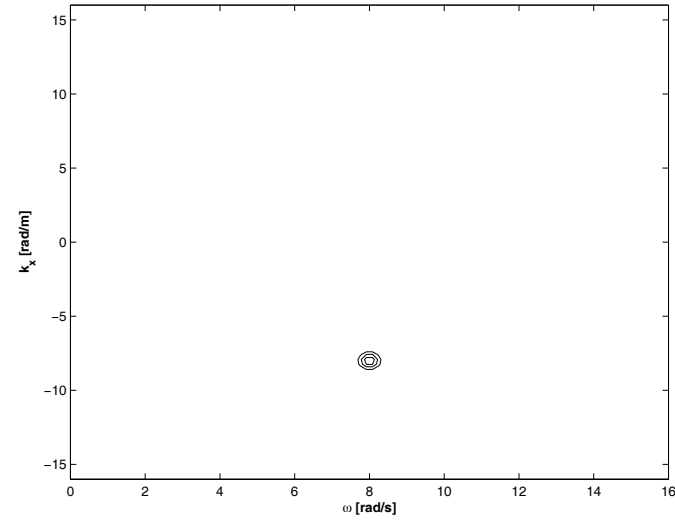

FIGURE 4: Filtered 2D FT.

\section{Analysis of 2D wave propagation}

The previous concept can be extended to the case of waves propagating in a $2 \mathrm{D}$ domain. We first consider a signal composed of a plain harmonic wave propagating in the $x>0$ direction, and of a secondary spherical wave generated at location $x_{s}, y_{s}$. The wave may be expressed as:

$$
w(x, y, t)=W_{0}\left[e^{i\left(\omega_{0} t-k_{x_{0}} x-k_{y_{0}} y\right)}+S e^{i\left(\omega_{0} t+k_{0} r-2 k_{0} r_{s}\right)}\right]
$$

where $W_{0}$ is the amplitude, $S$ is a generic scattering coefficient, and where $k_{x 0}=k_{0} \cos \theta$, $k_{y 0}=k_{0} \sin \theta, r_{s}=\left[x_{s}{ }^{2}+y_{s}{ }^{2}\right]^{1 / 2}$. The considered waveform can be represented in the frequency/wavenumber domain through the application of the 3D FT. Figure 5 shows the displacement distribution $w(x, y, t)$ over the considered domain at a specific instant of time, while Figure 6 shows a cross section of the magnitude of the 3D FT evaluated at frequency $\omega_{0}$. The plane wave component appears as a peak located at coordinates $k_{x}=-k_{x 0}$, $k_{y}=-k_{y}$, while the spherical wave appears as a semicircular contour limited to the $k_{x}>0$ half-plane. While the presence of the spherical wave as part of the considered displacement distribution is very evident in the wavenumber domain, it appears completely overshadowed by the plane wave component in the spatial displacement distribution of Figure 5. As discussed in the case of $1 \mathrm{D}$ wave propagation, the plane wave component can be conveniently removed through the simple application of a window function centered at the peak corresponding to the wavenumbers $k_{x}=-k_{x}, k_{y}=-k_{y}$, so that the spherical wave can be isolated and visualized separately from the overall measured response. The spatial distribution of the filtered signal is shown in Figure 7. The analysis of the residual signal displayed in Figure 7 allows the identification of location and potentially the characterization of the characteristics of the secondary wave source. 


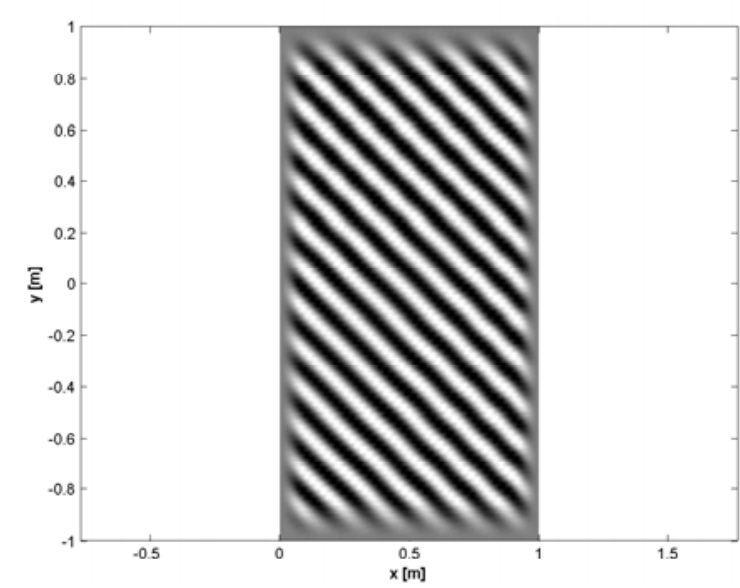

FIGURE 5: Snapshot of plane and spherical propagating wave.

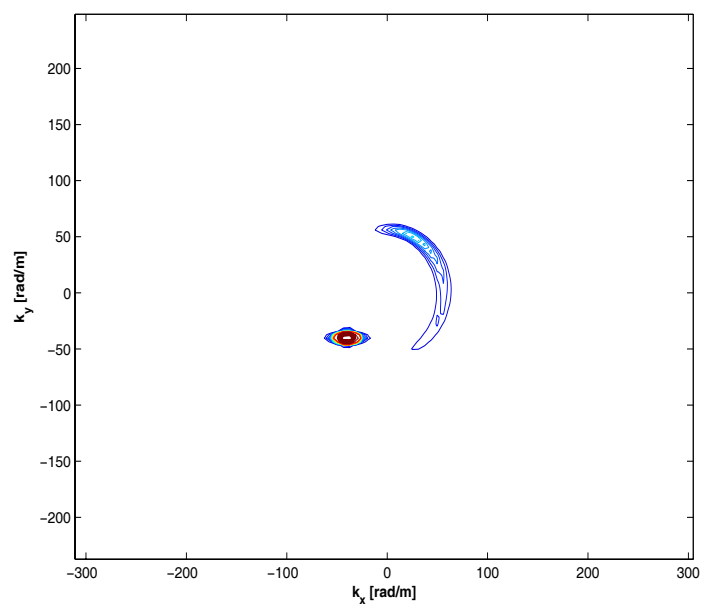

FIGURE 6: Cross section of 3D FT at $\omega_{0}$.

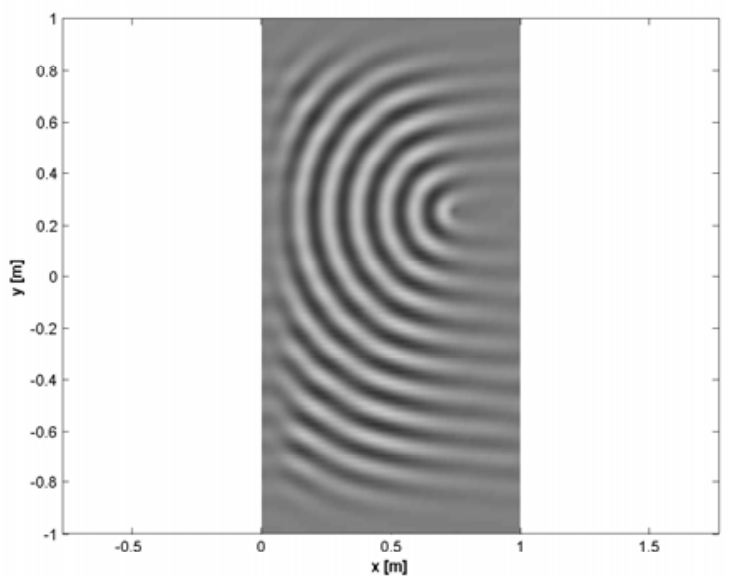

FIGURE 7: Filtered wave with removed plane wave component.

The residual signal as in the previous case, clearly shows the presence of the secondary wave and the location of its origin. In a practical setting, the excitation signal generated to interrogate the state of health of the structure can be considered as a primary wave, while the secondary wave can be associated to any damage or in general to any discontinuity in the structure. As the primary wave is selected for optimal excitation of the desired wave modes, one can consider it as fully known. As in the cases considered above, the nature of the filtering procedure can be selected on the basis of the type of excitation signal used for inspection.

\section{EXPERIMENTAL RESULTS}

\section{$\underline{\text { Set-up }}$}

The approach presented in the previous section is validated experimentally on simple plate structures, and on a tongue and grove bonded connection of the kind described in [9]. Piezoceramic discs are employed as actuators, and they are placed at locations which are selected so that elastic waves suitable for damage detection are generated [1,3]. The SLDV utilized (Polytec PI, Model PSV400M2) allows high 
frequency sampling $(\sim 1 \mathrm{MHz})$, which enables ultrasonic waves detection and visualization. In the wave propagation tests, the piezo discs are driven by a sinusoidal burst generated by a signal generator (Stanford Research Mod. SR360). The resulting elastic waves are recorded at the measurement grid points. The operation of the SLDV requires the generation of a pulse at each grid point in order to record the corresponding response. Phase information is retained by triggering the excitation signal through a low frequency signal $(10 \mathrm{~Hz})$, which also defines the scanning rate. Upon completion of measurements at all grid points, the recorded responses are then post-processed to obtain full images of the propagating wavefield within the region of inspection.

\section{Plate experiments}

A first set of experiments is performed on a simple aluminum plate artificially damaged at 4 locations. The plate measures $14 " \mathrm{x} 14$ " and it is 0.05 " thick. The considered damages are longitudinal slits of the same length and width, but varying depth, so that the sensitivity of the proposed technique with respect to increasing levels of damage can be evaluated. A schematic of the damaged configuration is given in Figure 8, while Table 1 provides details of the dimensions of the 4 longitudinal slits. The plate is excited by a piezoceramic disc of 0.6" of diameter and 0.030" of thickness (Steminc mod. SMD28T07F300). The actuator is placed approximately in the middle of the plate, equidistant from the 4 damages. The four regions of the plate surrounding the damages are scanned with the laser vibrometer. The excitation signal is a 5-cycle sinusoidal burst at $f_{0}=40 \mathrm{kHz}$. This frequency was selected after a manual sweep was performed in the 20-70 $\mathrm{kHz}$ range to evaluate the frequency of maximum response. The estimation of the optimal frequency of excitation based on a model of the piezoceramic disc bonded to the plate is currently under investigation and will support future developments of this study. The time response is transformed in the wavenumber frequency domain and is then filtered to isolate reflections according to the procedure outlined in the previous sections. The RMS map corresponding to the filtered time response is shown in Figure 9, which clearly highlights the location of 3 of the 4 damage sites, and provides indications of their extent through the intensity of the corresponding contour lines. No indication regarding the smallest damage is obtained through this representation. This limitation in sensitivity can be the result of noise in the data, or more likely can be associated to the relatively low excitation frequency used for the tests. These issues will be addressed in future refinements of the technique.

TABLE 1: Damage dimensions

\begin{tabular}{|r|r|r|r|}
\hline & $L_{D}$ & $w_{D}$ & $d_{D}$ \\
\hline 1 & $0.5 "$ & $0.05^{\prime \prime}$ & $0.03125 "$ \\
2 & $0.5 "$ & $0.05 "$ & $0.025 "$ \\
3 & $0.5 "$ & $0.05 "$ & $0.01875 "$ \\
4 & $0.5 "$ & $0.05 "$ & $0.00625 "$ \\
\hline
\end{tabular}




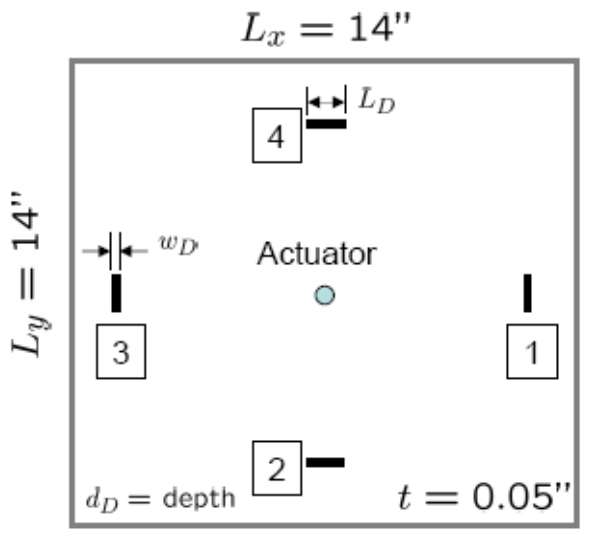

FIGURE 8: Schematic of damaged plate.

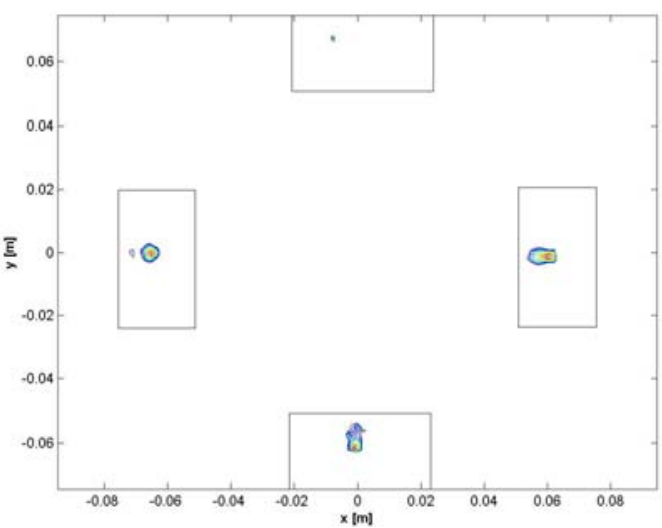

FIGURE 9: RMS of filtered response showing reflections from cracks.

\section{Inspection of a tongue\&grove bond}

Wave propagation tests were performed to investigate the effectiveness of the proposed technique to detect disbond in a tongue and grove bonded joint. The specimen considered for the experiments is shown in Figure 10, while the location of damage and the scanned region are displayed in Figure 11. The grove part of the joint was machined from an aluminum plate of thickness 3/8". The base plate thickness was kept at 0.1 " to allow a grove depth of $0.275 "$. Each grove side was machined to be $0.1 "$ thick. The grove width was $0.093 "$ while the tongue thickness was 0.081 " to allow for epoxy at the metal interface. A 1 " long section in the middle of the joint was coated with petroleum jelly to simulate a disbond, while the rest of the tongue and grove length was bonded using slow curing epoxy. The component is excited by a piezoceramic disc of 0.6 " of diameter and 0.030" of thickness (Steminc mod. SMD28T07F300) bonded on the inside surface of the test article. The SLDV measures the response of the specimen over an area selected across the joint. The specimen is excited by a 5-cycle sine burst at $125 \mathrm{KHz}$, frequency selected again after a manual sweep is performed across several frequencies. Results of the analysis are shown in Figure 11, which presents the RMS map of the filtered response and clearly shows the interaction between the propagating wave and the disbonded region. This technique could be attractive for testing of tongue and grove panels as it does not require their disassembly, and it allows scanning the front-side panel, while obtaining information regarding the bond quality. 


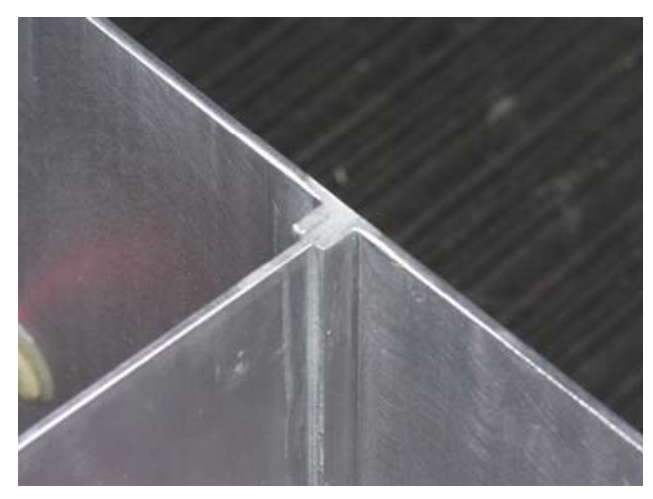

FIGURE 10 Tongue\&grove sample.

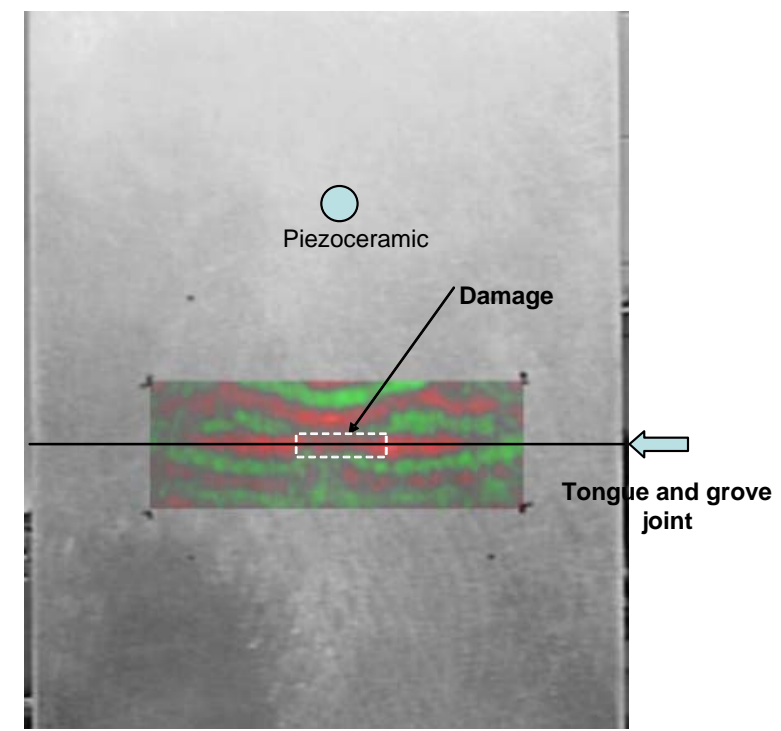

FIGURE 12: Scanned region and damage location.

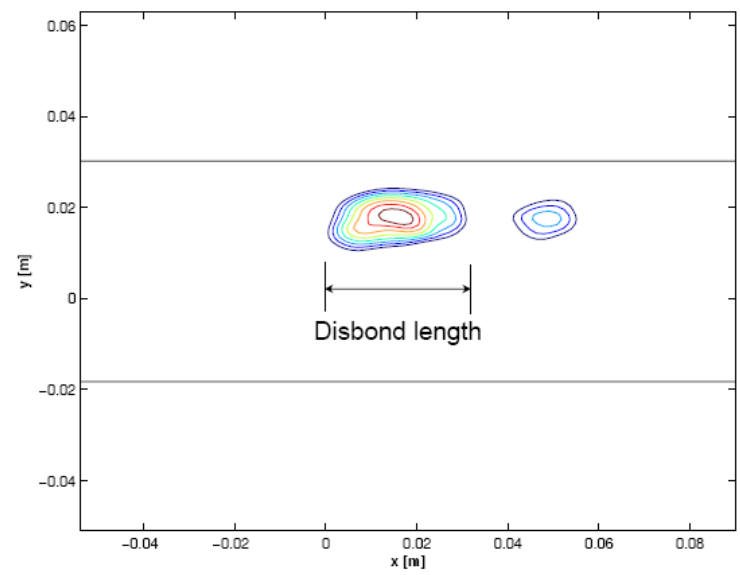

FIGURE 11: RMS of filtered response showing reflections caused by disbanded region.

\section{CONCLUSIONS}

This paper presents a filtering technique aimed at improving the damage visualization capabilities of full wavefield measurements. The technique operates in the frequency/wavenumber domain, where the presence of reflections associated to damage can be easily observed and separated from the main signal generated for inspection. Upon filtering, the residual signal highlights the location of the source of reflections and may be used for the characterization of the source itself. The technique is applied as part of a SHM technique based on guided wave inspection. The propagating waves are measured over a refined grid through a Scanning Laser Doppler Vibrometer which is able to provide the spatial and time resolution required to perform multidimensional Fourier Transforms. The application of the technique is first illustrated on a set of analytical and numerical examples and then it is successfully applied to analyze experimental data. The presented results demonstrate the potential of the technique and highlight future research needs. In particular, the technique needs to be supported by the optimal selection of frequency and mode of excitation, through methodologies that are currently under development. Moreover, the filtering process can be used to estimate the response of the undamaged 
component which can be used as a baseline for the formulation of damage indexes defining shape and size of the damage.

\section{REFERENCES}

1. Staszewski, W.J., Boller, C., Tomlinson, G., Health Monitoring Of Aerospace Structures. Smart Sensors And Signal Processing, Wiley\&Sons Ltd., 2004.

2. Rose, J.L. "A Baseline and Vision of Ultrasonic Guided Wave Inspection Potential" Journal of Pressure Vessel Technology, Vol. 124, pp. 273-282.

3. Giurgiutiu, V., "Tuned Lamb Wave Excitation and Detection With Piezoelectric Wafer Active Sensor of Structural Health Monitoring", Journal of Intelligent Material Systems and Structures, Vol. 16, No. 4, April 2005, pp. 291-305.

4. Wooh S.C., Shi Y., "Synthetic Phase Tuning of Guided Waves", IEEE Transactions on Ultrasonics, Ferroelectrics, and Frequency Control, Vol. 48, No. 1, January 2000, pp. 209-223.

5. Purekar A.S., Pines D.J., Sundararaman, S., Adams, D.E., "Directional Piezoelectric Phased Array Filters for Detecting Damage in Isotropic Plates", Smart Materials and Structures, 13(2004), pp. 838-850.

6. Sharma, V.K., Hanagud, S., Ruzzene, M., "Damage Index Estimation in Beams and Plates Using Laser Vibrometry", Proceeding of the 2005 International Workshop on Structural Health Monitoring (IWSHM), Stanford CA, September 2005.

7. Basri, R., Chiu, W.K., "Numerical analysis on the interaction of guided Lamb waves with a local elastic stiffness reduction in quasi-isotropic composite plate structures", Composite Structures, 66 (2004), pp. 87-99.

8. Alleyne, D., Cawley, P., "A Two-dimensional Fourier Transform Method for the Measurement of Propagating Multimode Signals", Journal of the Acoustical Society of America, Vol. 89, March 1991, pp. 1159-68.

9. http://www.aerostructures.goodrich.com/html. 